\title{
Dermal transfer quantification of nanoparticles from nano-enabled surfaces
}

Mackevica, Aiga; Olsson, Mikael Emil; Mines, Paul D.; Heggelund, Laura Roverskov; Foss Hansen, Steffen

Published in:

Nanolmpact

Link to article, DOI:

10.1016/j.impact.2018.06.001

Publication date:

2018

Document Version

Peer reviewed version

Link back to DTU Orbit

Citation $(A P A)$ :

Mackevica, A., Olsson, M. E., Mines, P. D., Heggelund, L. R., \& Foss Hansen, S. (2018). Dermal transfer quantification of nanoparticles from nano-enabled surfaces. Nanolmpact, 11, 109-118.

https://doi.org/10.1016/j.impact.2018.06.001

\section{General rights}

Copyright and moral rights for the publications made accessible in the public portal are retained by the authors and/or other copyright owners and it is a condition of accessing publications that users recognise and abide by the legal requirements associated with these rights.

- Users may download and print one copy of any publication from the public portal for the purpose of private study or research.

- You may not further distribute the material or use it for any profit-making activity or commercial gain

- You may freely distribute the URL identifying the publication in the public portal

If you believe that this document breaches copyright please contact us providing details, and we will remove access to the work immediately and investigate your claim 


\section{Accepted Manuscript}

Dermal transfer quantification of nanoparticles from nanoenabled surfaces

Nanolmpact

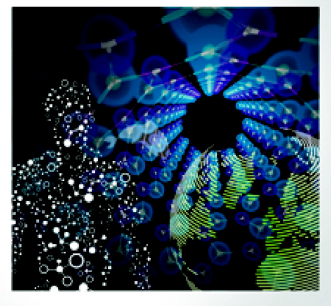

A. Mackevica, M.E. Olsson, P.D. Mines, L.R. Heggelund, S.F. Hansen

PII:

S2452-0748(18)30003-X

DOI: doi:10.1016/j.impact.2018.06.001

Reference:

IMPACT 121

To appear in:

NANOIMPACT

Received date:

5 January 2018

Revised date:

4 June 2018

Accepted date:

8 June 2018

Please cite this article as: A. Mackevica, M.E. Olsson, P.D. Mines, L.R. Heggelund, S.F. Hansen, Dermal transfer quantification of nanoparticles from nano-enabled surfaces. Impact (2017), doi:10.1016/j.impact.2018.06.001

This is a PDF file of an unedited manuscript that has been accepted for publication. As a service to our customers we are providing this early version of the manuscript. The manuscript will undergo copyediting, typesetting, and review of the resulting proof before it is published in its final form. Please note that during the production process errors may be discovered which could affect the content, and all legal disclaimers that apply to the journal pertain. 


\title{
Dermal transfer quantification of nanoparticles from nano-enabled
}

\section{surfaces}

A. Mackevica ${ }^{1}$, M.E. Olsson ${ }^{1}$, P.D. Mines ${ }^{2}$, L.R. Heggelund ${ }^{1}$, S.F. Hansen ${ }^{1}$

${ }^{1}$ Technical University of Denmark, Department of Environmental Engineering, Kgs. Lyngby, Denmark

${ }^{2}$ Technical University of Denmark, Department of Micro- and Nanotechnology, Kgs. Lyngby, Denmark

*Corresponding author - Technical University of Denmark, Department of Environmental Engineering, Bygningstorvet 115, DK - 2800 Kgs. Lyngby, Denmark, phone: +45 45251477, e-mail: aima@env.dtu.dk

\section{Graphical abstract}

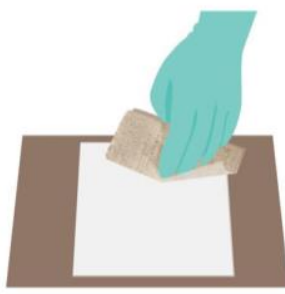

Wiping test

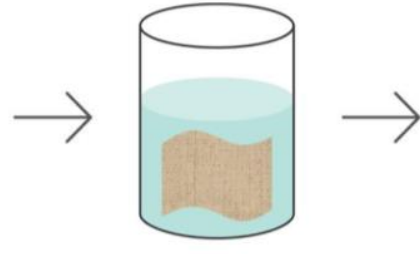

Particle extraction

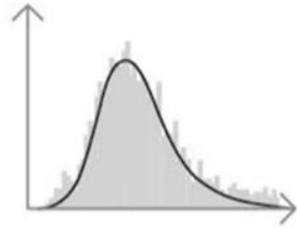

Size distribution Mass/number concentration

\begin{abstract}
Engineered nanoparticles are used in various applications due to their unique properties, which has led to their widespread use in consumer products. Silver, titanium, and copper-based nanoparticles (NPs) are a few of the commonly used nanomaterials in surface coatings, mainly due to their biocidal, optical, or photocatalytical properties. The knowledge concerning potential dermal exposure to nanoparticles from nanoparticle-enabled surfaces is currently lacking, partly due to analytical challenges. The aim of this study is to perform dermal wiping tests on nano-enabled surfaces and characterize NP release from keyboard covers and freshly painted surfaces, in terms of mass and number concentration, as well as released particle size distribution through the use of spICP-MS. Three types of NPs were selected for method validation testing, $\mathrm{Ag}, \mathrm{TiO}_{2}$, and $\mathrm{CuO}$; and, the particle extraction from wipes was found to be efficient for $\mathrm{Ag}$ and $\mathrm{CuO}$, but not for $\mathrm{TiO}_{2}$ particles. Thereafter, potential dermal transfer was tested by wipe sampling for two nanoAg-containing silicon keyboard covers, and wood painted with nanoCuO-containing paint. AgNP release was observed for one of the keyboard cover types, with around 5000 particles $/ \mathrm{cm}^{2}$ (corresponding to $0.002 \mathrm{ng} / \mathrm{cm}^{2}$ ) dislodged from the matrix after 3 wiping events. CuO NP release was 20000 particles $/ \mathrm{cm}^{2}\left(0.885 \mathrm{ng} / \mathrm{cm}^{2}\right)$ from the freshly painted surface, and magnitudes higher after the paint
\end{abstract}


was subjected to wear, reaching 1.4 million particles $/ \mathrm{cm}^{2}\left(2.5 \mathrm{ng} / \mathrm{cm}^{2}\right)$. The dermal transfer testing by wipe sampling and analytical approach used in this study demonstrates that wipe testing in combination with spICP-MS analysis can provide both qualitative data in terms of mass and number-based NP release, as well as particle characterization in terms of NP size distribution. Obtaining nano-specific release data can aid in providing a better understanding of dermal exposure to NPs from nano-enabled surfaces.

\section{Keywords}

Dermal exposure; release; nano-enabled surfaces; consumer products; wipe testing 


\section{Introduction}

The number of nano-enabled consumer products has been growing rapidly in recent years. There is still a high degree of uncertainty associated with the extent of exposure to nanoparticles (NPs) and the potential adverse effects exerted by NPs, which has led to an increased concern about both environmental and consumer safety (Hansen, 2017; Broomfield et al., 2017). There is a broad range of nano-enabled products available on the market, and many of them are likely to cause consumer exposure during normal use. According to the data presented in The Nanodatabase (2017), which holds an overview of what products that are marketed as "nano" are available on the EU market, when it comes to potential consumer exposure, dermal exposure is the most prominent route of exposure across most product categories (Hansen et al. 2016). Likelihood of consumer exposure to take place is attributed to both product properties and product use, the latter being highly dependent on how the user applies or uses the product.

Skin is the largest organ of the body and is a potential route of exposure through skin adsorption and subsequent inadvertent hand-to-mouth exposure. Nanoparticle penetration through skin has been shown to be dependent on skin integrity, as well as NP composition, shape and size, among other properties (Brouwer et al., 2016; Larese Filon et al., 2016; Oberdörster et al., 2005). A commentary by Larese Filon and coworkers based on the published literature with regard to NP penetration and penetration revealed that NPs smaller than $4 \mathrm{~nm}$ can penetrate and permeate intact skin, particles in sizes 4-20 nm can permeate both intact and damaged skin, 21-45 nm particles can penetrate and permeate only damaged skin, but NPs larger than $45 \mathrm{~nm}$ cannot penetrate nor permeate skin (Larese Filon et al., 2015).

Experimental studies dealing with dermal exposure to NPs are scarce, unless they are dealing with personal care products, such as sunscreens, which are widely known to contain different types of NPs (Lorenz et al., 2011). Much less attention has been drawn to assessing dermal exposure potential from nano-enabled coatings and paints, which are frequently in contact with human skin and may lead to inadvertent ingestion of NPs. Several experimental studies have investigated NP release from solid articles that may have potential for dermal exposure. Some of the examples include leaching from nano-enabled textiles either in wash water (e.g. Geranio et al. 2009; Lorenz et al. 2012; Impellitteri et al. 2009; Mitrano et al. 2014), or various types of artificial sweat (e.g. Geranio et al. 2009; von Goetz et al. 2013b), and the most commonly tested NP is Ag. Dermal transfer of NPs through simulated skin-surface contact has been addressed by a handful of studies (e.g. Platten et al., 2016; Quadros et al., 2013), which have used different wipes as a surrogate for human skin. Quadros et al. (2013) assessed potential dermal transfer from various baby products that were known to contain AgNPs, including plush toys, baby blankets, and disinfecting spray 
deposited on a surface. Surface wiping experiments revealed that there is a considerable potential for $\mathrm{Ag}$ transfer from product surfaces to the skin, ranging from 0.3 to $23 \mu \mathrm{gg} / \mathrm{m}^{2}$. Platten et al. (2016) investigated dermal exposure potential of copper particle pressure-treated lumber, also by using wipes as surrogate for skin exposure and found that around $1.5 \mathrm{mg} \mathrm{Cu} / \mathrm{m}^{2}$ was released per contact event. Studies like these provide relevant data for potential release from nano-enabled products that might result in dermal exposure, however, the dermal transfer is usually presented as the total amount of the chemical that is dislodged from the product, lacking nano/specific characterization for potential exposure to NPs.

As the nano-specific effects that might arise from NP exposure are not well known, characterization of the released particles is an essential part for understanding the total risks exerted by NPs. In this study, we aimed to perform dermal wiping tests on different nano-enabled surfaces and characterize NP release in terms of total mass concentration, particle number concentration, as well as released particle size distribution. We selected three types of NPs for testing $-\mathrm{Ag}, \mathrm{TiO}_{2}$, and $\mathrm{CuO}$, based on the knowledge about their applications for surfaces that may come into contact with skin and are thus relevant for dermal exposure testing. All three of those NPs are commonly used substances and additives in surface coatings $\mathrm{Ag}$ as an antibacterial coating for various food contact materials, textiles and personal care products, $\mathrm{TiO}_{2}$ in self-cleaning coatings due to its photocatalytical abilities for glass and ceramics, and $\mathrm{CuO}$ as an active biocidal substance in antifouling paints (Poland et al., 2013). The method for dermal transfer testing was following the NIOSH guideline Elements on Wipes: Method 9102 (NIOSH, 2003) with minor adjustments, namely, instead of acid-digesting the whole wipe for total contaminant analysis, the wipes were kept intact and particles were extracted without destroying the wipe. Before conducting the wiping tests, we performed method validation tests to establish a procedure for extracting particles from the wipes without destroying the sample and maximally maintaining the information regarding particle number and size. The NP characterization was performed by single particle inductively coupled plasma mass spectrometry (spICPMS). Thereafter, several NP-containing consumer products that are likely to come into contact with skin and therefore cause dermal exposure were selected for the execution of wiping tests, such as silicon keyboard covers coated with Ag NPs, and wooden blocks painted with antifouling paint containing CuO NPs. 


\section{Materials and methods}

\subsection{Nanoparticle suspensions and chemicals}

Three types of NP suspensions were used for method validation purposes in this study - citrate coated 30 $\mathrm{nm}$ Ag NP suspension (Cline, Sweden), 30-50nm $\mathrm{TiO}_{2}$ NP suspension (US Research Nanomaterials Houston, Texas, USA) and 30-50 nm CuO NP powder (PlasmaChem GmbH, Germany).

For the dermal transfer tests, artificial sweat was prepared according to ISO 105-E04 guideline (ISO, 2013) for alkaline sweat preparation. The composition of the artificial sweat was $0.5 \mathrm{~g} / \mathrm{L}$ L-histidine monohydrochloride monohydrate (99.99\%, Sigma Aldrich), $5 \mathrm{~g} / \mathrm{L}$ sodium chloride (>99.0\%, Sigma Aldrich), 5 $\mathrm{g} / \mathrm{L}$ disodium hydrogen orthophosphate dodecahydrate (>99.0\%, Sigma Aldrich), and $2.5 \mathrm{~g} / \mathrm{L}$ disodium hydrogen orthophosphate dehydrate (99.9\%, Merck). The final solution was adjusted to $\mathrm{pH} 8 \pm 0.2$ with 0.1 $\mathrm{mol} / \mathrm{L}$ sodium hydroxide solution.

\subsection{Consumer articles}

Several consumer articles with nano-enabled surfaces were selected for dermal transfer testing, namely two AgNP-enabled keyboard covers and one CuO NP-enabled acrylic paint on wood. Three types of commercially available silicon keyboard covers were purchased for testing of Ag NP transfer: two of those were advertising antimicrobial activity and claimed to contain AgNPs (referred to as Ag-1 and Ag-2), and one that was not advertising Ag content or antimicrobial activity was selected to be used as a Ag-free control (Ag-control). Products were purchased from online retailers (Amazon.de). The product information provided by the manufacturers and surface areas of the keyboard covers are noted in Table 1 . The Ag concentration in the keyboard covers was analyzed by ICP-MS (PerkinElmer, NexION 350D), following microwave-assisted acid digestion (Multiwave 3000, Anton-Paar). Samples were prepared by weighing 0.25 $\mathrm{g}$ of shredded keyboard covers and adding $5 \mathrm{~mL}$ of $\mathrm{HNO}_{3}$ (65\%, Merck) and $1 \mathrm{~mL}$ of $\mathrm{HF}$ (40\%, Merck). Thereafter, samples were microwave-digested at $1400 \mathrm{~W}$ for $15 \mathrm{~min}$. After digestion, $6 \mathrm{~mL} \mathrm{of} \mathrm{H}_{3} \mathrm{BrO}_{3}(10 \%$, Sigma Aldrich) were added for HF complexation, and samples were again microwaved at $600 \mathrm{~W}$ for $15 \mathrm{~min}$. Finally, samples were diluted with deionized (DI) water (18.2 M $\Omega$, Milli-Q, Merck KGaA) and analyzed using ICP-MS.

For testing of CuO NP transfer to the wipes, painted wooden blocks were used with sizes of $35 \times 35 \times 11$ $\mathrm{mm}$ (provided by BASF, Germany). The blocks were covered with CuO-containing acrylic paint on all sides with final $\mathrm{CuO}$ content of approximately $1.5 \%$ in the dried paint (final concentration corresponding to $5.7 \pm$ 
$0.2 \mathrm{mg} \mathrm{CuO} \mathrm{per} \mathrm{wooden} \mathrm{block)} \mathrm{(samples} \mathrm{henceforth} \mathrm{referred} \mathrm{to} \mathrm{as} \mathrm{CuO-paint).} \mathrm{The} \mathrm{CuO}$ embedded in paint were spherical with pristine particle sizes of $30-50 \mathrm{~nm}$, according to the information provided by the manufacturer. Blocks covered with the same type of CuO-free acrylic paint were used as control samples (CuO-control).

To obtain information regarding surface morphology and location, chemical identity and size of NPs in the products, the surfaces of the articles were analyzed by Scanning Electron Microscopy (SEM) (FEI, Quanta 200 ESEM FEG) imaging coupled with Energy-Dispersive X-Ray Spectroscopy (EDS) (Oxford Instruments 80 $\mathrm{mm}^{2} \mathrm{X}$-Max silicon drift detector).

The AgNP-enabled keyboard cover samples (Ag-1 and Ag-2) were prepared by cutting square pieces of the covers (approx. $0.5 \mathrm{~cm}^{2}$ ) and mounting them onto aluminum SEM specimen holders (Agar Scientific) using double-coated carbon conductive tape. Samples were then analyzed by SEM operated in high vacuum mode at an accelerating voltage of $10 \mathrm{KeV}$. To confirm the presence of silver, qualitative EDS was performed and analyzed using Aztec Software (Oxford Instruments).

To analyze the surface morphology of the painted wood samples, specimens were prepared by cutting triangular pieces of approximately $1 \mathrm{~cm}$ width from the corner of the wooden block by using a fine hacksaw. Each specimen was fixed onto aluminum specimen holders (Agar scientific) with carbon adhesive and strips of aluminum tape. Before analysis the specimens were surface coated with carbon for $3 \times 30$ seconds using a Carbon coater (Cressington, model 208).

The painted wood specimens were analyzed in low or high vacuum mode with acceleration voltages between $3-15 \mathrm{KeV}$. To confirm the presence of copper, qualitative EDS analysis was performed on selected specimens. 
Table 1: Description of the products used for wiping tests.

\begin{tabular}{|c|c|c|c|c|c|}
\hline & $\begin{array}{l}\text { Surface } \\
\text { area }\left(\mathrm{cm}^{2}\right)\end{array}$ & $\begin{array}{l}\text { Area used for } \\
\text { wiping }\left(\mathrm{cm}^{2}\right)\end{array}$ & $\begin{array}{l}\text { Mass per unit } \\
\text { area }\left(\mathrm{g} / \mathrm{cm}^{2}\right)\end{array}$ & $\begin{array}{l}\text { Product } \\
\text { information* }\end{array}$ & $\begin{array}{l}\text { Concentration } \\
\left(\mu \mathrm{g} / \mathrm{cm}^{2}\right)\end{array}$ \\
\hline \multicolumn{6}{|c|}{ Silicon keyboard covers } \\
\hline Ag-1 & 371 & 247 & 0.051 & Contains nano-Ag & 0.22 \\
\hline Ag-2 & 314 & 209 & 0.067 & Contains nano-Ag & 0.14 \\
\hline Ag-control & 285 & 190 & 0.049 & $\mathrm{n} / \mathrm{a}$ & $<0.08$ \\
\hline CuO-paint & 12 & 49 & 0.417 & CuO added & $9.5^{*}$ \\
\hline CuO-control & 12 & 49 & 0.417 & No CuO added & $\mathrm{n} / \mathrm{a} *$ \\
\hline
\end{tabular}

*Information provided by the manufacturer.

\subsection{Design and execution of wiping experiments}

As noted above, three types of keyboard covers were selected, as well as two types of painted wooden blocks to execute wiping experiments. The testing procedure for dermal transfer from the coated surfaces was following the NIOSH guideline Elements on Wipes: Method 9102 (NIOSH, 2003). Briefly, textile wipes were used as a surrogate for skin to test for dermal transfer from solid surfaces through dermal contact. Stepwise procedure is schematically represented in Figure 1. Three types of wipes were used for the wipe testing - (i) $100 \%$ ecological cotton for Ag testing, (ii) eco-labelled blend of $48 \%$ wool, $47 \%$ cotton and $5 \%$ polyamide for $\mathrm{CuO}$ testing, and (iii) $100 \%$ linen for $\mathrm{TiO}_{2}$ testing. More detailed information about these fabrics can be found elsewhere (referred to as samples A, B and C, respectively in Mackevica et al. 2018). The wipes were cut in $5 \times 5 \mathrm{~cm}$ size, and each wipe was wetted with $0.5 \mathrm{~mL}$ of artificial sweat $(\mathrm{pH} 8 \pm 0.2)$ before executing the wiping test. Then, the surface of the article was wiped by hand, using two fingertips held together and applying firm pressure, using an overlapping " $S$ " pattern covering the entire surface with horizontal strokes. Subsequently, the same wipe was used for wiping the same surface with overlapping "S" patterns with vertical strokes, and then once more with horizontal strokes. The wipe was then put in a polypropylene tube (Falcon $50 \mathrm{~mL}$ Conical Centrifuge Tubes) with $20 \mathrm{~mL}$ DI water, which was then sonicated in the sonicator bath (Retsch, UR 1) for $10 \mathrm{~min}$ and immediately analyzed by single particle ICPMS (spICP-MS). Further throughout the paper, this procedure is referred to as one wiping event. This procedure was repeated three times (i.e. three wiping events) on each surface to test for potential dermal transfer after multiple uses. The wiping procedure was executed on a laboratory weighing scale, in order to ensure that similar pressure was exerted during all wiping events (approximately $500 \mathrm{~g} / \mathrm{cm}^{2}$, which is similar to the force exerted by a person sitting on a chair, according to Clausen et al. (2016)). Because of the size of the weighing scale, size of keyboard cover samples had to be adjusted to the confined area that could fit on the scale. As the provided wooden block samples were relatively small and did not provide sufficient surface area for wiping tests, four blocks were put together to make a composite sample, creating a larger surface area that can be used for performing wiping events. For each wiping event, a new pair of 
gloves was used to avoid any carry-over and contamination. Three wipe samples were used as method blanks.

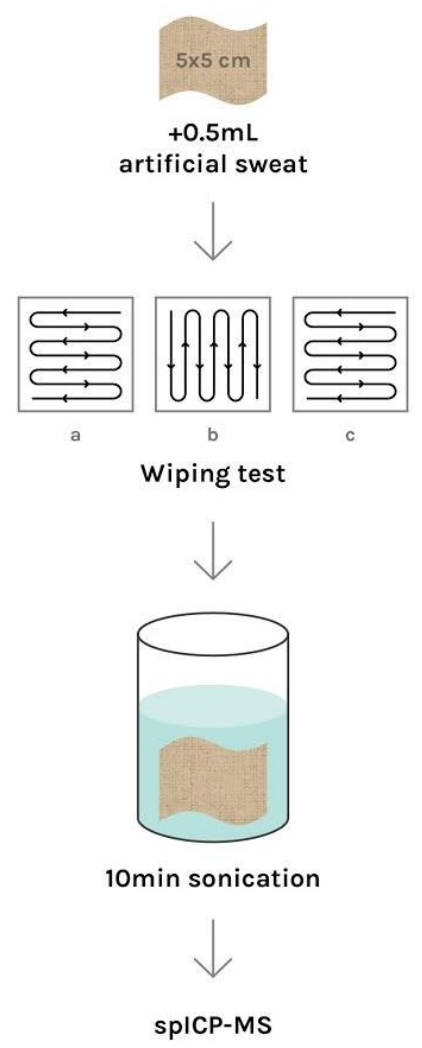

Figure 1: Schematic representation of execution of wiping tests, the procedure depicted here is referred to as one wiping event.

\subsection{Simulated wear of wooden blocks}

To simulate the wear of the CuO-paint coated wooden block samples, they were sanded by hand with 180 grit sand paper three times after the final wipe sampling event. While standardized sanding procedures usually include a Taber abrasion apparatus (e.g. ASTM, 2013; ASTM, 2014), we chose a simplified nonstandardized approach, namely sanding by hand, to simulate accelerated wear and tear of the surface. The sanding was done by applying the same procedure as for the wiping tests - by applying the sand paper with overlapping " $\mathrm{S}$ " patterns three times on the same surface. The pressure applied during sanding was the same as during wiping events, i.e. around $500 \mathrm{~g} / \mathrm{cm}^{2}$, which falls within typical pressure levels applied through Taber abrasion to mimic normal stress (15-500 kPa) applied to surfaces in a domestic setting, e.g. handling various furnishings (Shandilya et al. 2015). After sanding, the surface was subjected to the same wiping procedure as described above. 


\subsection{Characterization of nanoparticles}

The released NP content was measured by spICP-MS. Samples were diluted with DI water, if necessary, and Triton X-100 (0.0001\%) was added to the $\mathrm{TiO}_{2}$-containing samples. The instrument settings for spICP-MS measurements are presented in Table 2. The particle size was calculated based on the dissolved calibration curve, which was matrix-matched with the experimental samples (i.e. having the same content of artificial sweat). The transport efficiency was calculated using $60 \mathrm{~nm}$ Au particles (Perkin Elmer, USA), and the measurements were conducted in the same matrix as experimental samples. To evaluate the linear range of particle number concentration measurements, serial dilutions of NP suspensions were prepared to acquire the maximum particle number count for reliable and quantifiable measurements. The nanoparticle suspensions were sonicated by using a bath sonicator between every dilution step and also prior to the analysis by spICP-MS.

Table 2: spICP-MS operating parameters.

\begin{tabular}{llll}
\hline & $\mathrm{Ag}$ & $\mathrm{TiO}_{2}$ & $\mathrm{CuO}$ \\
\hline Gas flow $(\mathrm{mL} / \mathrm{min})$ & No gas & No gas & $5.7(\mathrm{He})$ \\
\hline Measurement time $(\mathrm{s})$ & 100 & 100 & 100 \\
\hline Dwell time $(\mu \mathrm{s})$ & 100 & 100 & 100 \\
\hline Analytes & ${ }^{48} \mathrm{Ti}^{44} \mathrm{Ca}$ & ${ }^{63} \mathrm{Cu}$ \\
\hline Mass fraction & $\mathrm{Ag}$ & 0.60 & 0.80 \\
\hline Density $\left(\mathrm{g} / \mathrm{cm}^{3}\right)$ & 1.00 & 4.23 & 6.31 \\
\hline Transport efficiency $(\%)$ & 10.49 & 7.59 & 8.74 \\
\hline Sample uptake rate $(\mathrm{mL} / \mathrm{min})$ & 8.89 & 0.303 & 0.307 \\
\hline LOD $_{\text {size }}(\mathrm{nm})$ & 0.304 & 30 & 28 \\
\hline
\end{tabular}

The lower size limit of detection ( $\left(\mathrm{OD}_{\text {size }}\right.$ ) for spICP-MS is defined as the lowest detectable particle diameter. It can usually be calculated by using the average background intensity (blank sample) and adding a value of three standard deviations and calculating the corresponding particle size (Pace et al., 2011). In this study, the $L O D_{\text {size }}$ was calculated based on the average of reported mean particle size for blank samples and adding three standard deviations.

As mentioned earlier, bigger particles might not be able to reach the plasma or be fully ionized in the plasma and thus result in inaccurate size calculation. Bigger particles and agglomerates also greatly contribute to the total mass calculations as well as shifting the mean particle size and introducing a higher degree of uncertainty for nanoparticle content in the sample. To obtain more robust results and set the focus on nanoscale measurements, only the particles up to $200 \mathrm{~nm}$ in size were counted as particle events. Particles and agglomerates above $200 \mathrm{~nm}$ were not included in particle number calculations as well as total particle mass fraction calculations. 
Transmission Electron Microscopy (TEM) was used for imaging of the particles released from the wipe samples. Approximately $4 \mu \mathrm{L}$ of the sample was transferred to a copper (for Ag-containing samples) or nickel (for Cu-containing samples) grid covered with holey carbon film (Agar Scientific, UK) and dried. TEM (FEI, Tecnai G2) was operated at $200 \mathrm{keV}$ accelerating voltage and the chemical identity of the particles was investigated by EDS. There was one replicate taken from each sample type.

\subsection{Recovery of nanoparticle extraction from wipes}

The nanoparticle extraction from the wipes is not a standardized procedure and to verify the applicability of the method to dermal transfer testing, particle recovery analysis was performed before executing the

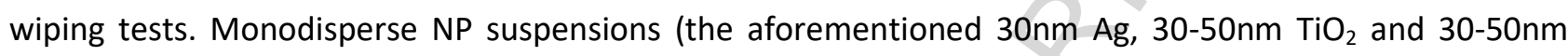
CuO NP suspensions) were used to spike the wipes, and then the particles were extracted in the exact same manner as in the wiping tests (described in 2.3 Design and execution of wiping experiments).

The schematic illustration of the recovery testing setup is shown in Figure 2. A $5 \times 5 \mathrm{~cm}$ wipe was wetted with $0.5 \mathrm{~mL}$ of artificial sweat, and then $0.25 \mathrm{~mL}$ of NP suspension was added (referred to as Sample). Then, the wipe was immersed into $20 \mathrm{~mL}$ deionized water in a polypropylene Falcon conical centrifuge tube, which was then sonicated for $10 \mathrm{~min}$ in a sonicator bath with added ice (to prevent particle dissolution). The suspension was then immediately analyzed by SpICP-MS, details of the analysis are provided in Table 1. To examine the quantity of NPs that can be extracted from the wipes, the same amount of NP suspension and artificial sweat was added to $20 \mathrm{~mL}$ of DI water and treated the same way (NP-control). To check for the background created by the wipe itself, wipes were wetted with $0.5 \mathrm{~mL}$ of artificial sweat and immersed in $20 \mathrm{~mL}$ DI water (Control). The mixture of DI water and artificial sweat was used as a blank sample (Blank). The method validation was then based on the differences between the recovered NP amounts from the different samples. All samples were prepared in triplicates. 


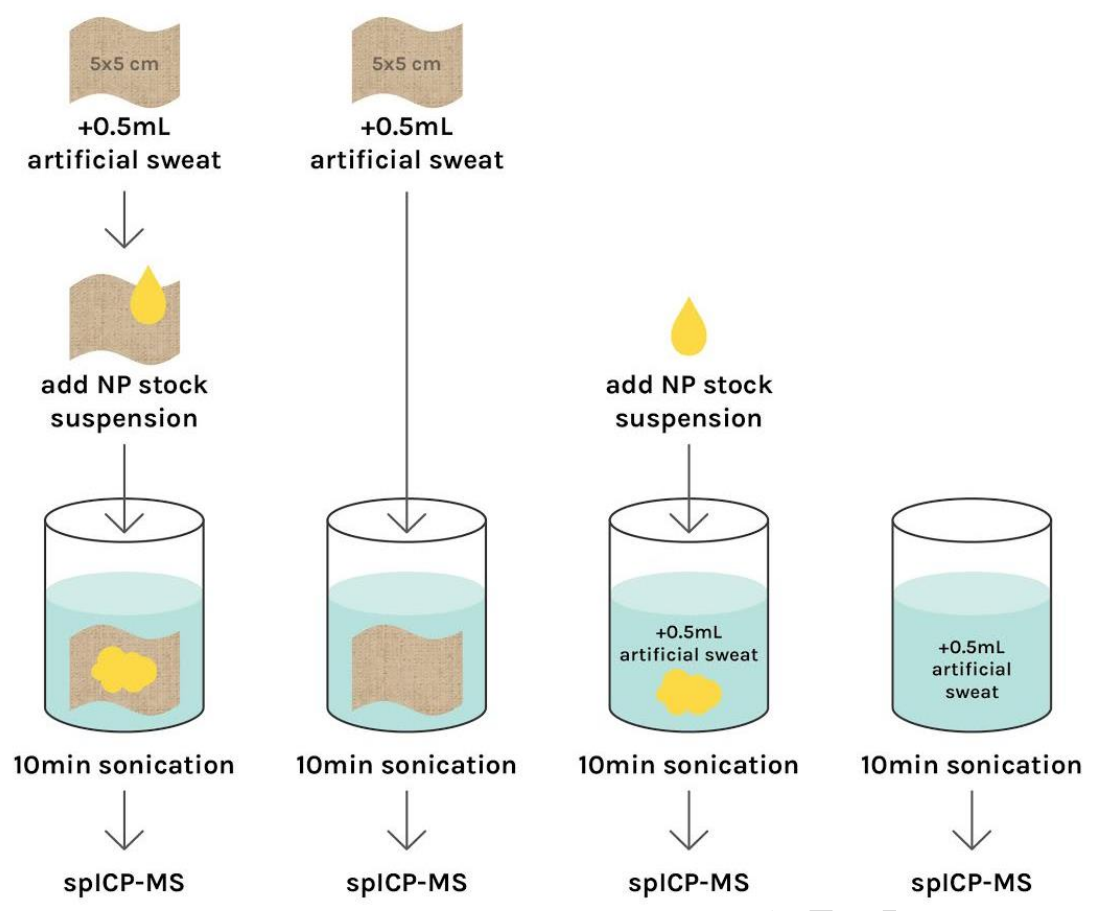

Figure 2: Schematic representation of the setup used for testing of NP extraction efficiency from the wipes. From left to right: Sample, Control, NP-control, Blank. 


\section{Results and discussion}

\subsection{Method validation for NP extraction from the wipes}

Before executing the wiping tests, method validation tests were performed to evaluate the applicability of the particle extraction process for the actual wiping experiments. Three types of NPs were selected for testing, namely $\mathrm{Ag}, \mathrm{TiO}_{2}$ and $\mathrm{CuO}$, and the method validation was done by spiking the wipes with NP suspensions and simulating the wiping test conditions. Amongst the three NP types, Ag and CuO exhibited relatively high recoveries in terms of NP size and particle number that can be extracted from the wipes. For particle number concentration, $81 \%$ of Ag NPs could be extracted by this method, and $84 \%$ of CuO NPs, while $\mathrm{TiO}_{2}$ had a recovery of only $6 \%$, indicating that particles likely remain on the textile fibers and ultrasonication is not able to dislodge them from the textile matrix. In terms of mass concentration recovery of NPs, Ag recovery was $82 \%$, while for $\mathrm{CuO}$ and $\mathrm{TiO}_{2}$ it was $109 \%$ and $36 \%$, respectively. For CuO the extracted NP mass concentration was demonstrated to be slightly overestimated, indicating that the wipe could contribute to the increase in CuO background (Figure S1, Supplementary Information), and some $\mathrm{CuO}$ particles could be aggregating or agglomerating, leading to larger particle size (Table 3 ) and fewer particle events, thus lowering particle number concentration. CuO data for the blank (DI water and artificial sweat) showed relatively high particle counts, but this did not contribute significantly to the total mass of $\mathrm{CuO}$ recovered. It might be an indication for counting "false positives", i.e. smaller particles that likely are part of the background signal rather than represent actual CuO particles.

Considering particle size measurements, it can be observed that the extraction process had minor effect on $\mathrm{Ag}$ and $\mathrm{CuO}$ particle sizes. As depicted in Table 3, AgNP sizes were very close to the nominal particle size indicated by the manufacturer, and both mean and mode sizes remained almost entirely unchanged after extraction from the wipes. Similarly, CuO did not exhibit large deviations from pristine particle size, however, nearly $20 \%$ increase was observed for both mean and mode sizes of NPs after extraction process. The dissolved fraction for all NP types was below the limit of detection $(<0.5 \mu \mathrm{g} / \mathrm{L})$.

For method validation of $\mathrm{TiO}_{2} \mathrm{NPs}$, the extraction process was unsuccessful. Pristine particles in diluted artificial sweat medium resulted in slightly higher particle size measurements than the nominal size of the particles (60 nm instead of 30-50 nm range). The reason might be $\mathrm{TiO}_{2}$ interactions with the artificial sweat components present in the medium, or the agglomeration of the particles. It has been shown before that stability and the size of $\mathrm{TiO}_{2}$ particles in suspension are highly dependent on the $\mathrm{pH}$ and ionic strength of the medium (Cupi et al., 2016). In low pH and lower ionic strength media $\mathrm{TiO}_{2} \mathrm{NPs}$ are able to form stable aggregates of fewer particles, whereas increased ionic strength, in this case as a result of the relatively high $\mathrm{NaCl}$ content in the artificial sweat medium, facilitates formation of larger aggregates. 
Table 3: spICP-MS size measurements for method validation of NP extraction from the wipes. Average size \pm standard deviation is presented for the measured data, $n=3$. NP-control - DI water and artificial sweat medium spiked with NPs; Sample - NPs extracted in DI water from wipe wetted with artificial sweat and spiked with NPs.

\begin{tabular}{llllll}
\hline & $\begin{array}{l}\text { Nominal size } \\
(\mathrm{nm})\end{array}$ & \multicolumn{2}{l}{$\begin{array}{l}\text { Measured mean size } \\
(\mathrm{nm})\end{array}$} & \multicolumn{2}{l}{$\begin{array}{l}\text { Measured mode size } \\
(\mathrm{nm})\end{array}$} \\
\cline { 3 - 6 } & & $\begin{array}{l}\text { NP- } \\
\text { control }\end{array}$ & Sample & $\begin{array}{l}\text { NP- } \\
\text { control }\end{array}$ & Sample \\
\hline $\mathrm{Ag}$ & 30 & $31 \pm 0$ & $31 \pm 1$ & $29 \pm 0$ & $28 \pm 1$ \\
\hline $\mathrm{TiO}_{2}$ & $30-50$ & $72 \pm 1$ & $178 \pm 4$ & $60 \pm 1$ & $132 \pm 3$ \\
\hline $\mathrm{CuO}$ & $30-50$ & $53 \pm 2$ & $62 \pm 1$ & $41 \pm 1$ & $49 \pm 1$ \\
\hline
\end{tabular}

Based on the data from method validation testing, it was concluded that this type of NP extraction method works well for AgNPs and is valid for quantification of dermal transfer of AgNPs from surfaces to wipes. For CuO extraction the method can be used for quantitative measurements, but the results have to be interpreted with caution, taking into account the slight overestimation in particle size and mass recovery after the extraction procedure. $\mathrm{TiO}_{2}$ measurements indicated that this setup is not suited for testing dermal transfer of $\mathrm{TiO}_{2} \mathrm{NPs}$, due to the very poor particle extraction efficacy. Other extraction methods might be more suited to determine the quantity of NPs transferred to the wipes, for instance, methods that would allow degradation of the wipe matrix without interfering with the size distribution and number concentration of NPs. It has already been done with other types of NPs in different organic matrices, such as such as using enzymatic digestion to separate AgNPs from chicken meat (Loeschner et al., 2015), or employing solvent extraction for Ag- and AuNP in Daphnia magna (Gray et al., 2013) and $\mathrm{TiO}_{2}$ in sunscreens (Nischwitz et al., 2012). For NPs that are insoluble in acids, which includes $\mathrm{TiO}_{2}$, acid digestion with e.g. nitric acid could be applicable for matrix degradation (Laborda et al., 2016), and it has been successfully utilized for determination of $\mathrm{SiO}_{2}$ in tomato soup (Wagner et al., 2015) and biological matrices (Tadjiki et al., 2009). Moreover, different sampling methods may be more suited for $\mathrm{TiO}_{2}$ transfer testing, such as tape stripping, patch sampling, or hand washing, provided that study allows the use of volunteers (Schneider et al., 2000). 


\subsection{Characterization of the articles}

Consumer products that were selected for wiping tests were wooden blocks coated with $\mathrm{CuO}$ containing paint, and two types of keyboard covers that claimed to contain AgNPs. The total NP content for $\mathrm{CuO}$ in the wooden blocks was provided by the manufacturer and corresponded to 9.5 $\mu \mathrm{g} / \mathrm{cm}^{2}$, whereas $\mathrm{Ag}$ content in the keyboard covers was experimentally determined by microwaveassisted acid digestion and ICP-MS analysis and resulted in 0.22 and $0.14 \mu \mathrm{g} / \mathrm{cm}^{2}$ for Ag-1 and Ag-2, respectively (Table 1 ).

Surface characterization, including both surface morphology and elemental analysis with EDS, was performed on samples of Ag-1 and Ag-2, and CuO-paint using SEM. Ag-1 exhibited an amorphous landscape with very few pronounced defects and almost no discernible particles not labelled as silicon with EDS, making individual nanoparticle recognition extremely difficult (Figure 3a, 3b). Although, when a region of approx. $400 \mu^{2}$ was analysed with EDS, a small signal of Ag was detected, corresponding to $<0.1 \% \mathrm{Ag}$ (Figure S2, Supplementary Information). Ag-2, on the other hand, displayed a highly patterned morphology with many waves and ridges creating a uniform texture throughout consisting of primarily silicon (Figure 3c, 3d). Again, as was the case with Ag-1, when a region of approx. $400 \mu \mathrm{m}^{2}$ was analysed with EDS, a small signal of Ag was detected, corresponding to $<0.1 \%$ $\mathrm{Ag}$ (Figure S3, Supplementary Information). These Ag quantities in Ag-1 and Ag-2, however, are not surprising given the extremely low amount of Ag detected with ICP-MS.

CUO-paint samples were analysed by SEM-EDS before and after performing the sanding procedure. The surface of unused and unmodified samples (Figure $4 \mathrm{a}$ and $4 \mathrm{~b}$ ) had a grainy structure and larger agglomerates forming the surface, where the EDS analysis confirmed the presence of copper and titanium on the surface of the unused specimen (Figure S4, Supplementary Information). Copper only seems to be present in very low amounts, with EDS signal corresponding to $<0.1 \%$, whereas titanium signals were detected in relatively high amounts. Titanium is used as a pigment for the paint, and the abundance of Ti particles on the painted surface was making it more difficult to detect individual CuO particles that might be present on the surface. The low magnification SEM images confirmed the influence of sanding treatment on the surface morphology (Figure $4 b$ and $4 c$ ), having scratches on the surface that are exposing deeper layers of paint, which may facilitate particle release and further surface degradation. 

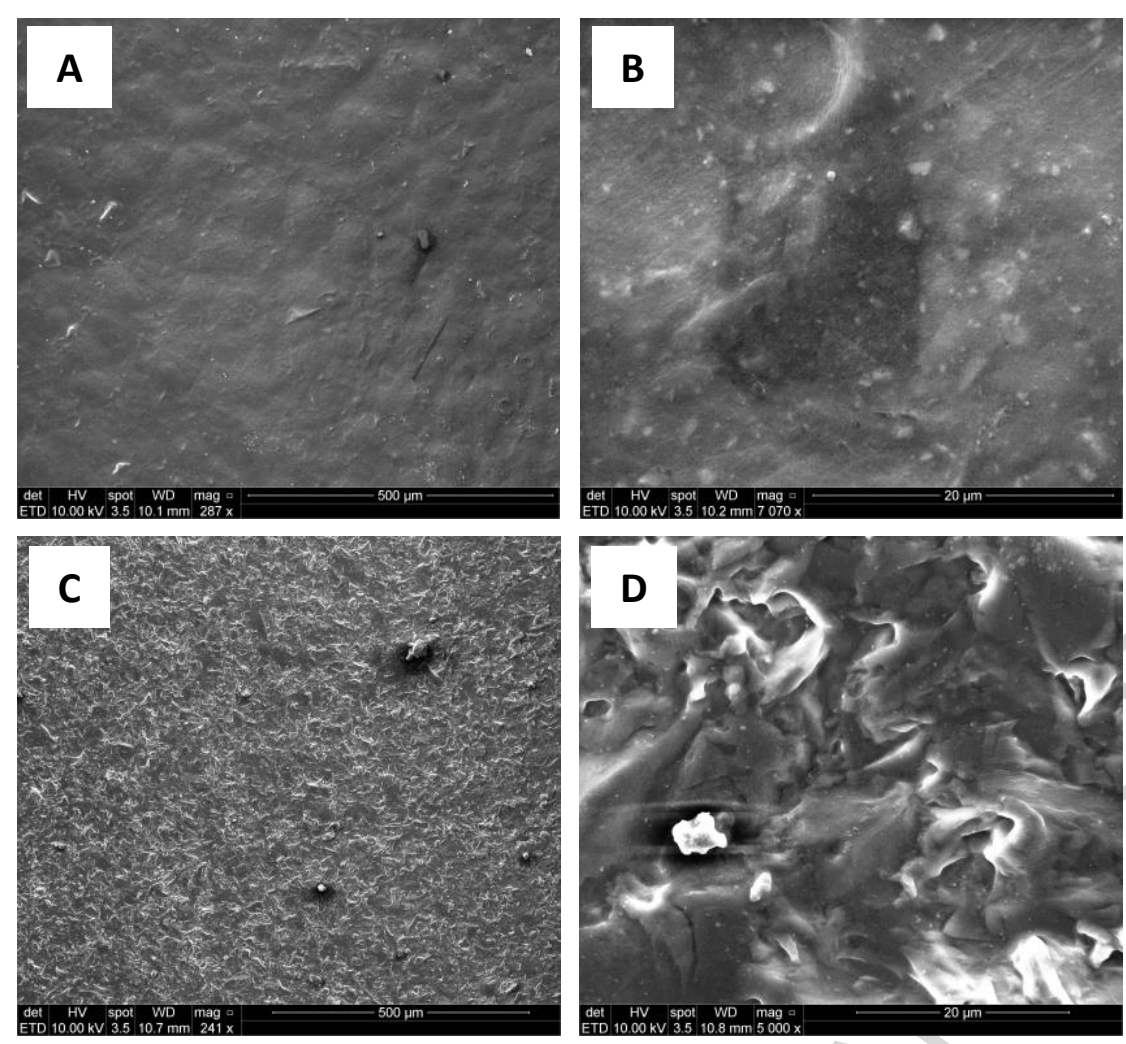

Figure 3: SEM images of keyboard covers in various magnifications. A and B: Ag-1 sample, C and D: Ag-2 sample.
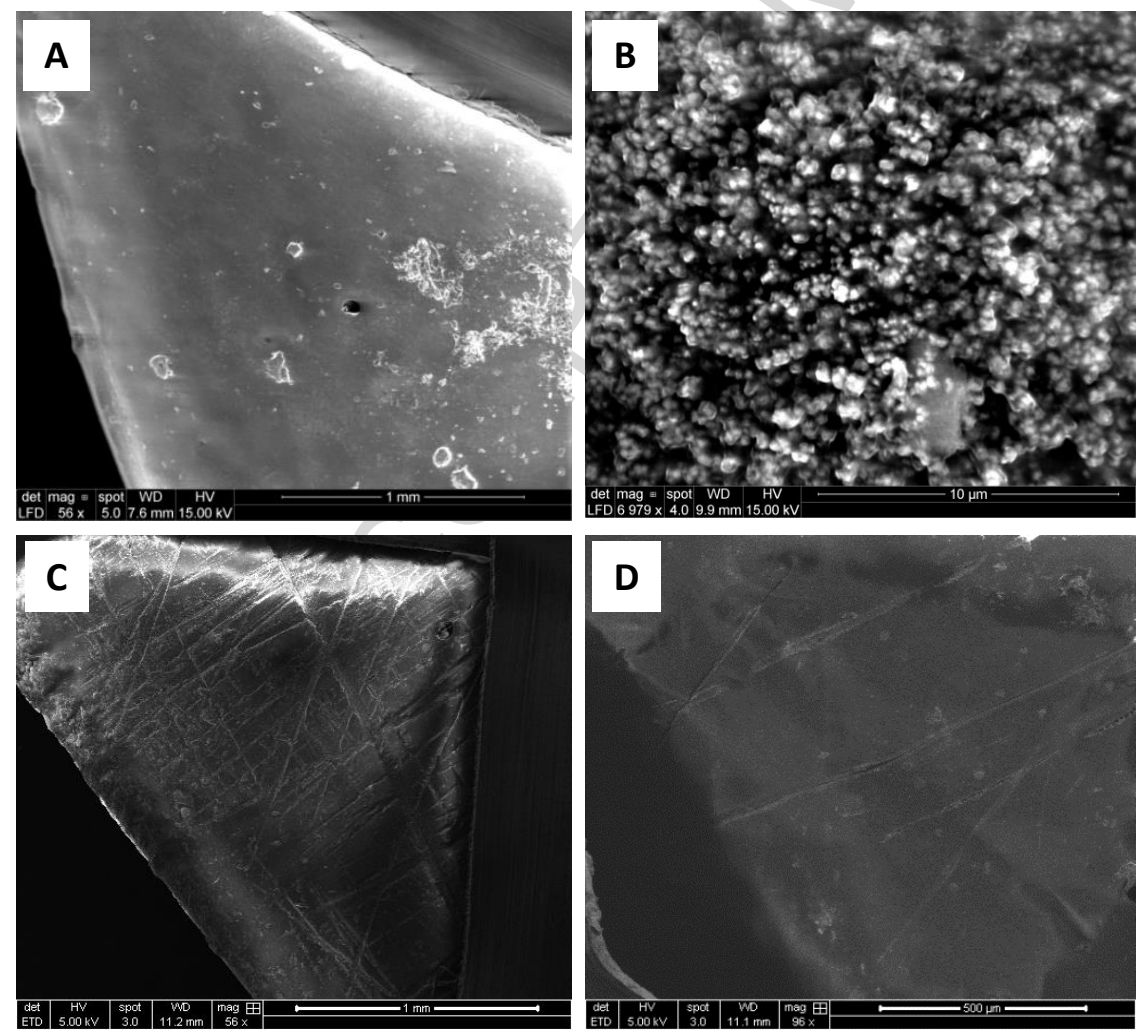

Figure 4: SEM images of wooden blocks painted with CUO-containing paint before and after sanding procedure. A and B: CuO-paint sample, C and D: CuO-paint sample after sanding. 


\subsection{Silver nanoparticle transfer from keyboard covers}

From the three types of commercial keyboard covers tested, only one of the types (Ag-2) showed Ag NP release that was notably different from the control keyboard covers (Ag-control) (Figure 5). For all three sample types the AgNP transfer could be considered rather negligible, as the total mass release is in sub-ng per $\mathrm{cm}^{2}$ range and very close to the limit of detection. Furthermore, no AgNPs were identified by TEM-EDS due to the very low NP concentrations in the test suspensions. As analyzed by SpICP-MS, the wiping test for Ag-2 keyboard resulted in a maximum nanoparticle transfer of $0.001 \mathrm{ng} / \mathrm{cm}^{2}$, corresponding to around 2000 particles $/ \mathrm{cm}^{2}$. The mean size for particles released from Ag-2 keyboard covers was $28 \mathrm{~nm}$, and for Ag1 and Ag-control keyboard covers the mean released particle sizes were 27 and $25 \mathrm{~nm}$, respectively (Table S1, size distributions presented in Figure S6, Supplementary Information). The dermal loading from these articles may be considered negligible, as the magnitude of Ag released is low, also in comparison with other items that have been tested for dermal transfer potential. For example, dermal wiping study by Quadros et al. (2013) addressed surface transfer from such commercial Ag NP-enabled items as baby blanket, plush toy, dried disinfecting spray, dry surface wipes and kitchen scrubber. Their tests resulted in silver transfer of $0.03 \mathrm{ng} / \mathrm{cm}^{2}$ for kitchen scrubber, and the highest transfer of $2.3 \mathrm{ng} / \mathrm{cm}^{2}$ measured for baby blanket. Even for the highest observed releases, the authors concluded that the amounts of Ag released are below the threshold that could cause damage even to one of the most sensitive populations at risk, such as children. However, it must be noted that only the total Ag release was reported and the characterization of Ag NP release was lacking.

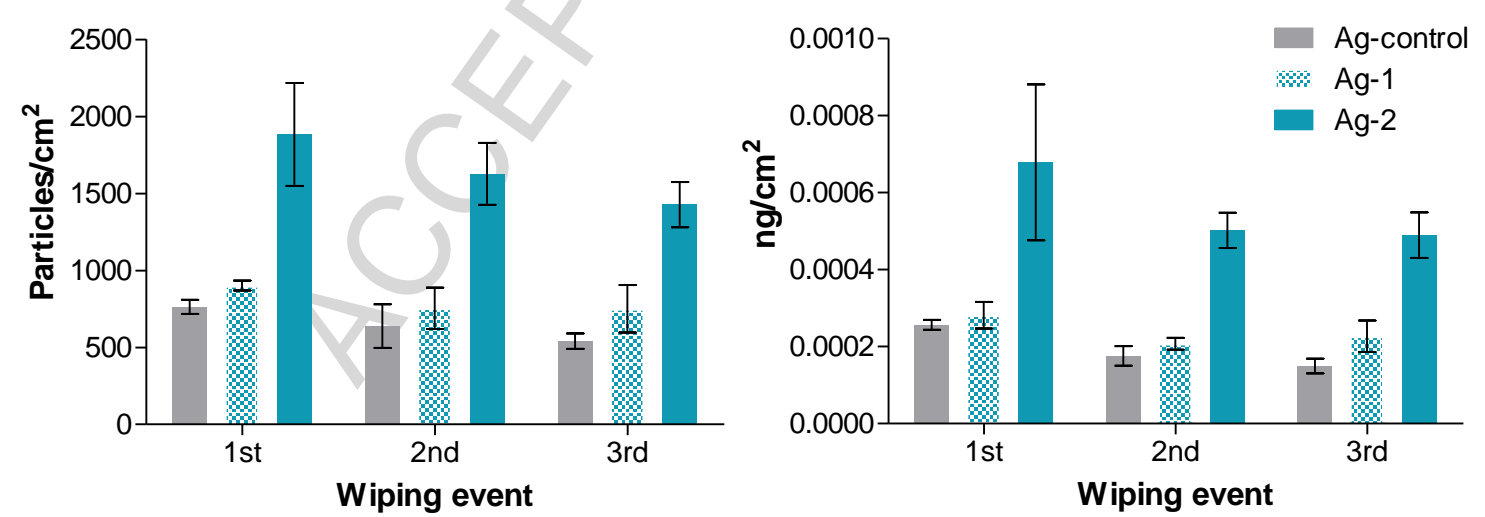

Figure 5: Ag NP transfer from keyboard covers to wipes. Left - transfer in number of particles per $\mathrm{cm}^{2}$, Right - mass transfer in $\mathrm{ng} / \mathrm{cm}^{2}$. Error bars represent standard error of mean, $\mathrm{n}=3$.

A general trend can be observed that AgNP transfer is decreasing with the subsequent wiping events, indicating that most likely with increasing frequency of use the Ag NPs would detach from the surface until 
no more transfer is possible. Several studies have reported that surface-bound NPs have a tendency to show high initial release, and then slower subsequent release rates with each following use of the same article. For example, several food contact materials with Ag NPs in the inner lining have shown declining release after multiple uses (Echegoyen and Nerín, 2013; von Goetz et al., 2013a). Silver NP coatings for textiles have also shown the same pattern, where most Ag was released after already two washing events (e.g. Limpiteeprakan et al. 2016). Generally, desorption of NPs is assumed to be dependent on several important factors, such as how strongly the particle is bound to the matrix of the article, as well as how the product itself is treated by the consumer. Release of NPs through desorption is more likely when NPs are loosely bound to the surface of the product, which is the case for e.g. spray-on coatings. If NPs are embedded in the matrix, the NP release is dependent on how the product is treated, i.e. if matrix degradation can be induced which would allow liberation of particles from the solid (Duncan and Pillai, 2015). In the case of Ag-enabled keyboard covers, it can be assumed that fraction of the particles are present as surface coating, but there might be AgNPs that are also relatively strongly bound to or embedded in the silicon matrix, as there is continuous release observed by wiping tests.

\subsection{Copper oxide nanoparticle transfer from painted wooden blocks}

Dermal transfer testing for the painted wooden blocks showed that the CuO NP release from painted and sanded wooden blocks was markedly different from blocks with untreated paint. Sanding by hand was used to simulate accelerated wear and tear of the painted surface, to depict potential dermal exposure from older paint. While this type of sanding procedure arguably provides less reproducibility than standardized Taber abrasion methods, the applied pressure used in this study is similar to normal stress levels applied to surfaces in a domestic setting, as described in section 2.4.

In terms of released particle counts, there was nearly no CuO NP release from the CuO-containing paint matrix for the paint that had not been subjected to any weathering or external impacts, and the observed release was not significantly different from CuO-control samples (Figure 6). However, after sanding of the paint surface, the $\mathrm{CuO}$ release was substantially increasing and the released $\mathrm{Cu}$ concentrations were magnitudes higher. Various types of natural and artificial weathering and aging of paints are known to have an effect on NP release, e.g. UV exposure increasing $\mathrm{TiO}_{2}$ release from painted surfaces (Olabarrieta et al., 2012), milling and aging facilitating $\mathrm{SiO}_{2}$ release from paints (Al-Kattan et al., 2015), or accelerated aging by sanding resulting in elevated amounts of micronized Cu dislodging from treated wood (Platten et al., 2016).

In the current study, particle count was markedly increased when wiping the sanded wooden blocks, resulting in potential dermal transfer of up to $5 \cdot 10^{5}$ particles per $\mathrm{cm}^{2}$, compared to around $2 \cdot 10^{5}$ particles 
per $\mathrm{cm}^{2}$ for non-sanded paint (observed in the $2^{\text {nd }}$ wiping event for Cu-paint samples, see Figure 6). The mean sizes of the released particles were $84 \mathrm{~nm}$ and $79 \mathrm{~nm}$ for CuO-paint without and with sanding, respectively, and the mode sizes were $61 \mathrm{~nm}$ and $54 \mathrm{~nm}$ without and with sanding, respectively (Table S1, size distributions presented in Figure S7, Supplementary Information). The total mass of CuO released after three wiping events was 0.9 and $2.5 \mathrm{ng} / \mathrm{cm}^{2}$ for non-sanded and sanded paint, respectively, which is less than $0.01 \%$ of the $\mathrm{CuO}$ amount originally present in the painted surface per $\mathrm{cm}^{2}$.

The sizes of the released NPs are similar to the size of the pristine CuO particles (presented in Table 3), that were used for painting the wooden blocks, according to the manufacturer. This might indicate that high amount of un-altered particles can be released from the paint matrix, especially after some initial wearand-tear, weathering or long-term normal use. TEM imaging, however, revealed that bigger agglomerates are also released by wiping, in sizes up to $200 \mathrm{~nm}$ (Figure 7). Freely occurring single particles were not found by TEM imaging, but that may also be because of drying artifacts during sample preparation for TEM analysis, e.g. drying. Even if larger agglomerates are observed by TEM, it may be that they are broken up and dispersed as single particles or smaller agglomerates during ultrasonication allowing spICP-MS to detect suspended individual particles.
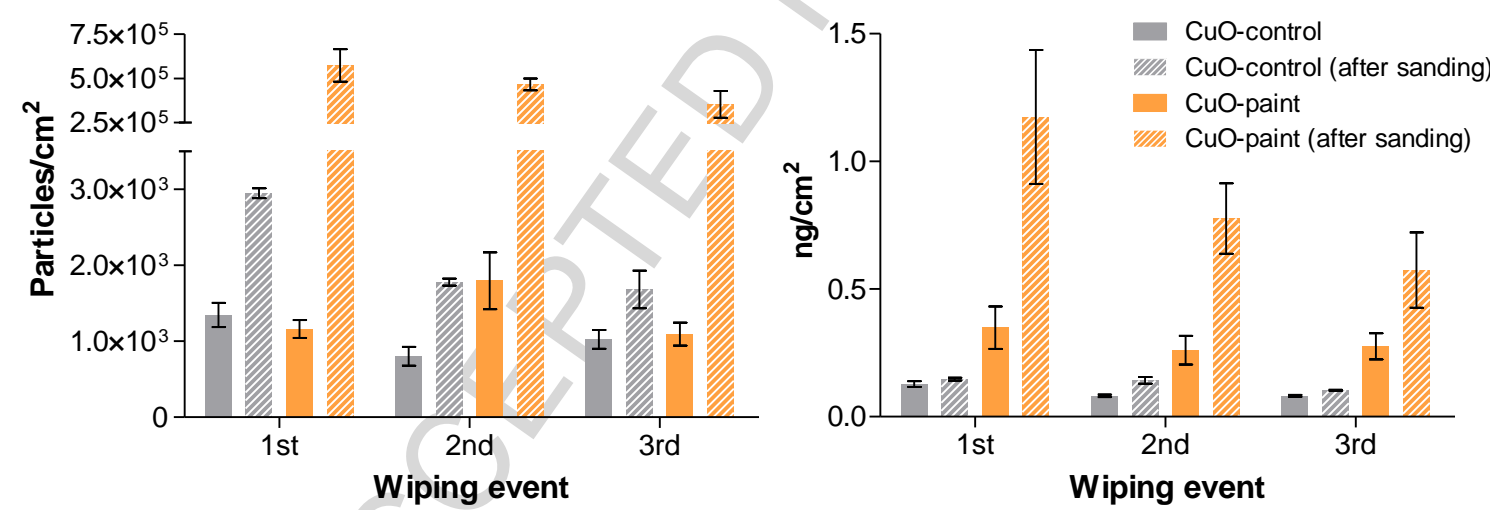

Figure 6: CuO NP transfer from painted wooden blocks to wipes. Left - transfer in number of particles per $\mathrm{cm}^{2}$, Right mass transfer in $\mathrm{ng} / \mathrm{cm}^{2}$. Error bars represent standard error of mean, $\mathrm{n}=3$.

The potential dermal transfer from painted surfaces has not been extensively addressed in the literature so far. Only a handful of studies have addressed this, including a study regarding dermal transfer of Cu from pressure-treated lumber, which was focusing on micronized and ionic copper azole-treated wood (Platten et al., 2016). This study was applying a dermal wiping method developed by Consumer Product Safety Commission (Thomas et al., 2005), which involves, in brief, using a wiping apparatus that applies controlled pressure on a confined area. The total released $\mathrm{Cu}$ was extracted from the wipes by acid digestion followed by ICP-AAS analysis, and revealed no significant differences in total $\mathrm{Cu}$ release between timber that was 
impregnated with micronized or ionized $\mathrm{Cu}$. A total of 12 wiping events showed that there was higher initial release of $\mathrm{Cu}$ from micronized $\mathrm{Cu}$-treated timber during the first three wiping events, whereafer the release was reaching a plateau. Additionally, the wood that was previously weathered exhibited considerably higher total $\mathrm{Cu}$ release quantities up to around $25 \mathrm{mg} \mathrm{Cu} / \mathrm{m}^{2}$ for the first wiping event after wood exposure to weathering. The release of other types of $\mathrm{NPs}$, such as $\mathrm{Cu}_{2} \mathrm{O}, \mathrm{TiO}_{2}$, and $\mathrm{Ag}$, from painted surfaces has been investigated by several other studies (e.g. Adeleye et al., 2016; Kaegi et al., 2010, 2008; Olabarrieta et al., 2012), which have shown that weathering or abrasion are important factors for dislodging NPs from the paint matrix, and NP release can be magnitudes higher when the paint is not intact and unused.
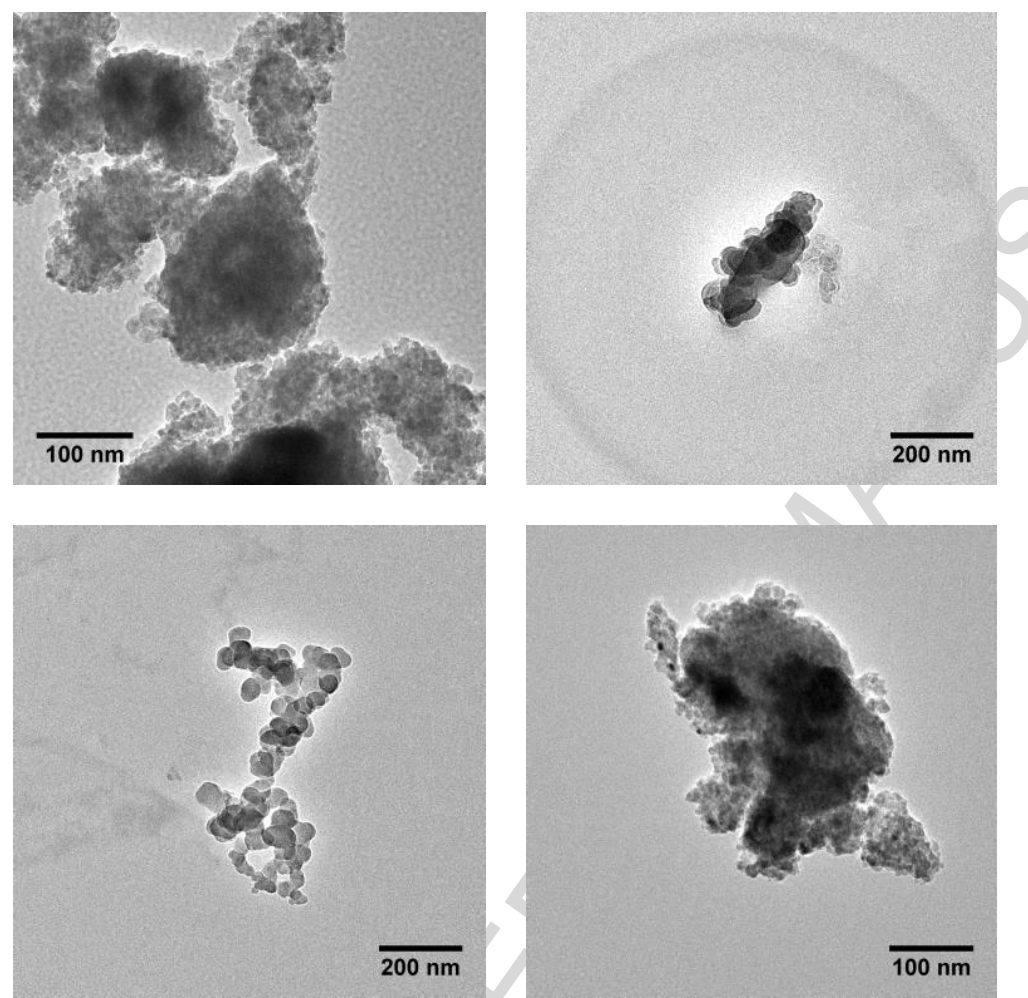

Figure 7: TEM images from particles released from CuO-paint sample ( $2^{\text {nd }}$ Wiping event).

Nano $\mathrm{CuO}$ is known to be more toxic than micronized $\mathrm{CuO}$ particles, and the assumed mechanism of toxicity is the release of $\mathrm{Cu}^{2+}$ ions that leads to intracellular reactive oxygen species (ROS) generation (Keller et al., 2017). Generally, dermal exposure to copper compounds is not known to be of concern apart from potential allergic reactions. However, also ingestion of copper has been shown to have toxic effects (Civardi et al., 2015; Platten et al., 2016). Hand-to-mouth exposure particularly might be of concern when it comes to Cu-treated surfaces that come into contact with skin. The study by Thomas et al. (2005) attempted to estimate what would be possible child exposure to wood preservatives. It was estimated that the area of treated lumber surfaces that a child might come into contact with during a typical visit to a playground is $1.29 \mathrm{~m}^{2}$. Considering the highest CuO release observed in the present study ( $1^{\text {st }}$ wiping event after sanding 
of CuO-paint samples), it can be estimated that assuming this area for exposure, a child might have up to $0.15 \mathrm{mg} \mathrm{CuO}$ in total transferred to the hand. 


\section{Conclusions}

In this study, we investigated the potential for dermal transfer of nanoparticles from nano-enabled solid surfaces, namely two commercially available Ag NP-containing silicon keyboard covers, and industrially manufactured CuO NP-containing paint that was applied on wooden blocks. The dermal transfer of Ag and CuO particles was quantified by using spICP-MS, and additionally, released particles were characterized by TEM-EDS. Initial method validation for dermal transfer measurements was conducted for three nanomaterial types, $\mathrm{Ag}, \mathrm{TiO}_{2}$ and $\mathrm{CuO}$, by spiking the wipes with the NPs and extracting them via ultrasonication. Results obtained by spICP-MS determined that ultrasonication of the wipes can extract around $81 \%$ of the Ag nanoparticles transferred to the wipes and $84 \%$ of the CuO NPs, with little or no changes in particle size due to the extraction process. It was concluded that this method is not applicable for $\mathrm{TiO}_{2}$ particles, due to poor extraction efficiency (around 6\%). For analysis of $\mathrm{CuO}$ and Ag NP dermal transfer from solid surfaces, the analytical approach presented in this paper provides a relatively quick procedure for NP extraction and analysis, which again provides both qualitative data in terms of mass and number-based release, as well as particle characterization in terms of NP size distribution. Additional measurements using other analytical techniques would be necessary to investigate potential transformation of the particles (e.g. complexation, oxidation, dissolution).

Sampling of transfer from the Ag keyboard covers demonstrated relatively low released amounts, both in terms of particle number and mass concentration. Only one of two types had a higher release compared to the control sample, with the total release resulting in only $<0.1 \%$ of the initial Ag content. The analysis of $\mathrm{CuO}$ transfer from the painted surface to the wipes indicated that CuO NPs could indeed be dislodged from the painted surface, both when paint was intact or subjected to wear and tear. After simulated surface wear of the paint (i.e. sanding by hand), the observed release of CuO NPs was magnitudes higher in terms of particle counts, but after three consecutive wiping events less than $0.01 \%$ of the initial CuO was released.

\section{Acknowledgements}

This project has received funding from the European Union's Seventh Framework Programme [FP7/20072013] under EC-GA No. 604305 'SUN'. We sincerely thank our lab technicians Sinh Hy Nguyen and Susanne Kruse for all their help during this project, as well as Marika Latsone for the help with the graphic design. 


\section{References}

Adeleye, A.S., Oranu, E.A., Tao, M., Keller, A.A., 2016. Release and detection of nanosized copper from a commercial antifouling paint. Water Res. 102, 374-382. doi:10.1016/j.watres.2016.06.056

Al-Kattan, A., Wichser, A., Vonbank, R., Brunner, S., Ulrich, A., Zuin, S., Arroyo, Y., Golanski, L., Nowack, B., 2015. Characterization of materials released into water from paint containing nano-SiO2. Chemosphere 119, 1314-1321.

doi:10.1016/j.chemosphere.2014.02.005

ASTM, 2013. Standard Test Method for Resistance of Transparent Plastics to Surface Abrasion, ASTM D1044-13. ASTM International, West Conshohocken, PA.

ASTM, 2014. Standard Test Method for Abrasion Resistance of Organic Coatings by the Taber Abraser, ASTM D4060-14. ASTM International, West Conshohocken, PA.

Brouwer, D.H., Spaan, S., Roff, M., Sleeuwenhoek, A., Tuinman, I., Goede, H., van Duuren-Stuurman, B., Filon, F.L., Bello, D., Cherrie, J.W., 2016. Occupational dermal exposure to nanoparticles and nano-enabled products: Part 2, exploration of exposure processes and methods of assessment. Int. J. Hyg. Environ. Health 219, 503-512. doi:10.1016/j.ijheh.2016.05.003

Broomfield, M., Hansen, S.F., Pelsy, F. 2017. Support for $3^{\text {rd }}$ regulatory review of nanomaterials - environmental legislation. Brussels: European Commission.

Civardi, C., Schwarze, F.W.M.R., Wick, P., 2015. Micronized copper wood preservatives: An efficiency and potential health risk assessment for copper-based nanoparticles. Environ. Pollut. 200, 126-132. doi:10.1016/j.envpol.2015.02.018

Clausen, P.A., Spaan, S., Brouwer, D.H., Marquart, H., le Feber, M., Engel, R., Geerts, L., Jensen, K.A., Kofoed-Sørensen, V., Hansen, B., De Brouwere, K., 2016. Experimental estimation of migration and transfer of organic substances from consumer articles to cotton wipes: Evaluation of underlying mechanisms. J. Expo. Sci. Environ. Epidemiol. 26, 104-112. doi:10.1038/jes.2015.35

Cupi, D., Hartmann, N.B., Baun, A., 2016. Influence of pH and media composition on suspension stability of silver, zinc oxide, and titanium dioxide nanoparticles and immobilization of Daphnia magna under guideline testing conditions. Ecotoxicol. Environ. Saf. 127, 144-152. doi:10.1016/j.ecoenv.2015.12.028

Duncan, T. V., Pillai, K., 2015. Release of Engineered Nanomaterials from Polymer Nanocomposites: Diffusion, Dissolution, and Desorption. ACS Appl. Mater. Interfaces 7, 2-19. doi:10.1021/am5062745

Echegoyen, Y., Nerín, C., 2013. Nanoparticle release from nano-silver antimicrobial food containers. Food Chem. Toxicol. 62, 16-22. doi:10.1016/j.fct.2013.08.014

Hansen, S.F., 2017. React now regarding nanomaterial regulation. Nature nanotechnology, 12, p.714.

Hansen, S.F., Heggelund, L.R., Revilla Besora, P., Mackevica, A., Boldrin, A., Baun, A., 2016. Nanoproducts - what is actually available to European consumers? Environ. Sci. Nano 3, 169-180. doi:10.1039/C5EN00182J

Geranio, L., Heuberger, M., Nowack, B., 2009. The behavior of silver nanotextiles during washing. Environ. Sci. Technol. 43, 81138118.

Gray, E.P., Coleman, J.G., Bednar, A.J., Kennedy, A.J., Ranville, J.F., Higgins, C.P., 2013. Extraction and Analysis of Silver and Gold Nanoparticles from Biological Tissues Using Single Particle Inductively Coupled Plasma Mass Spectrometry. Environ. Sci. 
Technol. 47, 14315-14323. doi:10.1021/es403558c

Impellitteri, C.A., Tolaymat, T.M., Scheckel, K.G., 2009. The Speciation of Silver Nanoparticles in Antimicrobial Fabric Before and After Exposure to a Hypochlorite/Detergent Solution. J. Environ. Qual. 38, 1528. doi:10.2134/jeq2008.0390

ISO, 2013. ISO 105-E04:2013 - Textiles: Tests for colour fastness. Part E04: Colour fastness to perspiration.

Kaegi, R., Sinnet, B., Zuleeg, S., Hagendorfer, H., Mueller, E., Vonbank, R., Boller, M., Burkhardt, M., 2010. Release of silver nanoparticles from outdoor facades. Environ. Pollut. 158, 2900-2905. doi:10.1016/j.envpol.2010.06.009

Kaegi, R., Ulrich, A., Sinnet, B., Vonbank, R., Wichser, A., Zuleeg, S., Simmler, H., Brunner, S., Vonmont, H., Burkhardt, M., Boller, M., 2008. Synthetic TiO2 nanoparticle emission from exterior facades into the aquatic environment. Environ. Pollut. 156, 233239. doi:10.1016/j.envpol.2008.08.004

Keller, A.A., Adeleye, A.S., Conway, J.R., Garner, K.L., Zhao, L., Cherr, G.N., Hong, J., Gardea-Torresdey, J.L., Godwin, H.A., Hanna, S., Ji, Z., Kaweeteerawat, C., Lin, S., Lenihan, H.S., Miller, R.J., Nel, A.E., Peralta-Videa, J.R., Walker, S.L., Taylor, A.A., TorresDuarte, C., Zink, J.I., Zuverza-Mena, N., 2017. Comparative environmental fate and toxicity of copper nanomaterials. Nanolmpact 7, 28-40. doi:10.1016/j.impact.2017.05.003

Laborda, F., Bolea, E., Cepriá, G., Gómez, M.T., Jiménez, M.S., Pérez-Arantegui, J., Castillo, J.R., 2016. Detection, characterization and quantification of inorganic engineered nanomaterials: A review of techniques and methodological approaches for the analysis of complex samples. Anal. Chim. Acta 904, 10-32. doi:10.1016/j.aca.2015.11.008

Larese Filon, F., Bello, D., Cherrie, J.W., Sleeuwenhoek, A., Spaan, S., Brouwer, D.H., 2016. Occupational dermal exposure to nanoparticles and nano-enabled products: Part I-Factors affecting skin absorption. Int. J. Hyg. Environ. Health 219, 536544. doi:10.1016/j.ijheh.2016.05.009

Larese Filon, F., Mauro, M., Adami, G., Bovenzi, M., Crosera, M., 2015. Nanoparticles skin absorption: New aspects for a safety profile evaluation. Regul. Toxicol. Pharmacol. 72, 310-322. doi:10.1016/j.yrtph.2015.05.005

Limpiteeprakan, P., Babel, S., Lohwacharin, J., Takizawa, S., 2016. Release of silver nanoparticles from fabrics during the course of sequential washing. Environ. Sci. Pollut. Res. 1-9. doi:10.1007/s11356-016-7486-3

Loeschner, K., Navratilova, J., Grombe, R., Linsinger, T.P.J., Købler, C., Mølhave, K., Larsen, E.H., 2015. In-house validation of a method for determination of silver nanoparticles in chicken meat based on asymmetric flow field-flow fractionation and inductively coupled plasma mass spectrometric detection. Food Chem. 181, 78-84. doi:10.1016/j.foodchem.2015.02.033

Lorenz, C., Von Goetz, N., Scheringer, M., Wormuth, M., Hungerbühler, K., 2011. Potential exposure of German consumers to engineered nanoparticles in cosmetics and personal care products. Nanotoxicology 5, 12-29. doi:10.3109/17435390.2010.484554

Lorenz, C., Windler, L., Goetz, N. Von, Lehmann, R.P., Schuppler, M., Hungerbühler, K., Heuberger, M., Nowack, B., 2012. Chemosphere Characterization of silver release from commercially available functional ( nano ) textiles. Chemosphere 89, 817-824. doi:10.1016/j.chemosphere.2012.04.063

Mackevica, A., Olsson, M.E. and Hansen, S.F., 2018. Quantitative characterization of TiO 2 nanoparticle release from textiles by conventional and single particle ICP-MS. Journal of Nanoparticle Research, 20(1), p.6.

Mitrano, D., Rimmele, E., Wichser, A., Erni, R., Height, M., Nowack, B., 2014. Presence of nanoparticles in wash water from conventional silver and nano-silver textiles. ACS Nano 8, 7208-7219. doi:10.1021/nn502228w 
NIOSH, 2003. Elements on wipes: Method 9102, in: NIOSH Manual of Analytical Methods 4th Edition.

Nischwitz, V., Goenaga-Infante, H., Bolea, E., Castillo, J.R., Scherrers, R., Ludwig, C., Ulrich, A., Rose, J., Bottero, J.-Y., Zazueta, C., Pedraza-Chaverri, J., Garcia-Cuellar, C.M., Chirino, Y.I., 2012. Improved sample preparation and quality control for the characterisation of titanium dioxide nanoparticles in sunscreens using flow field flow fractionation on-line with inductively coupled plasma mass spectrometry. J. Anal. At. Spectrom. 27, 1084. doi:10.1039/c2ja10387g

Oberdörster, G., Maynard, A., Donaldson, K., Castranova, V., Fitzpatrick, J., Ausman, K., Carter, J., Karn, B., Kreyling, W., Lai, D., Olin, S., Monteiro-Riviere, N., Warheit, D., Yang, H., 2005. Principles for characterizing the potential human health effects from exposure to nanomaterials: elements of a screening strategy. Part. Fibre Toxicol. 2, 8. doi:10.1186/1743-8977-2-8

Olabarrieta, J., Zorita, S., Peña, I., Rioja, N., Monzón, O., Benguria, P., Scifo, L., 2012. Aging of photocatalytic coatings under a water flow: Long run performance and TiO2 nanoparticles release. Appl. Catal. B Environ. 123, 182-192. doi:10.1016/j.apcatb.2012.04.027

Pace, H.E., Rogers, N.J., Jarolimek, C., Coleman, V.A., Higgins, C.P., Ranville, J.F., 2011. Determining Transport Efficiency for the Purpose of Counting and Sizing Nanoparticles via Single Particle Inductively Coupled Plasma Mass Spectrometry. Anal. Chem. 83, 9361-9369. doi:10.1021/ac201952t

Platten, W.E., Sylvest, N., Warren, C., Arambewela, M., Harmon, S., Bradham, K., Rogers, K., Thomas, T., Luxton, T.P., 2016. Estimating dermal transfer of copper particles from the surfaces of pressure-treated lumber and implications for exposure. Sci. Total Environ. 548, 441-449. doi:10.1016/j.scitotenv.2015.12.108

Poland, C.A., Read, S.A.K., Varet, J., Carse, G., Christensen, F.M., Hankin, S.M., 2013. Dermal Absorption of Nanomaterials. Part of the "Better control of nano" initiative 2012-2015, Environmental Project No. 1504, 2013. Copenhagen, Denmark.

Quadros, M.E., Pierson, R., Tulve, N.S., Willis, R., Rogers, K., Thomas, T.A., Marr, L.C., 2013. Release of Silver from NanotechnologyBased Consumer Products for Children. Environ. Sci. Technol. 47, 8894-8901. doi:10.1021/es4015844

Schneider, T., Cherrie, J.W., Vermeulen, R., Kromhout, H., 2000. Dermal exposure assessment. Ann. Occup. Hyg. 44, $493-499$. doi:10.1093/annhyg/44.7.493

Shandilya, N., Le Bihan, O., Bressot, C. and Morgeneyer, M., 2015. Emission of titanium dioxide nanoparticles from building materials to the environment by wear and weather. Environmental science \& technology, 49(4), pp.2163-2170. DOI: 10.1021/es504710p

Tadjiki, S., Assemi, S., Deering, C.E., Veranth, J.M., Miller, J.D., 2009. Detection, separation, and quantification of unlabeled silica nanoparticles in biological media using sedimentation field-flow fractionation. J. Nanoparticle Res. 11, 981-988. doi:10.1007/s11051-008-9560-3

The Nanodatabase, 2017. The Nanodatabase [WWW Document]. URL http://nanodb.dk/ (accessed 3.1.17).

Thomas, T.A., Levenson, M.S., Cobb, D.G., Midgett, J.D., Porter, W.K., Saltzman, L.E., Bittner, P.M., 2005. The Development of a Standard Hand Method and Correlated Surrogate Method for Sampling CCA (Pressure)-Treated Wood Surfaces for Chemical Residue. J. Child. Heal. 2, 181-196. doi:10.3109/15417060490930047

von Goetz, N., Fabricius, L., Glaus, R., Weitbrecht, V., Günther, D., Hungerbühler, K., 2013a. Migration of silver from commercial plastic food containers and implications for consumer exposure assessment. Food Addit. Contam. Part A 30, 612-620. 
von Goetz, N., Lorenz, C., Windler, L., Nowack, B., Heuberger, M., Hungerbühler, K., 2013b. Migration of Ag- and TiO2-

(Nano)particles from Textiles into Artificial Sweat under Physical Stress: Experiments and Exposure Modeling. Environ. Sci. Technol. 47, 9979-9987.

Wagner, S., Legros, S., Loeschner, K., Liu, J., Navratilova, J., Grombe, R., Linsinger, T.P.J., Larsen, E.H., von der Kammer, F., Hofmann, T., Solans, C., Lehner, A., Allmaier, G., 2015. First steps towards a generic sample preparation scheme for inorganic engineered nanoparticles in a complex matrix for detection, characterization, and quantification by asymmetric flow-field flow fractionation coupled to multi-angle light scattering and ICP-MS. J. Anal. At. Spectrom. 30, 1286-1296. doi:10.1039/C4JA00471J 


\section{Highlights}

- Potential transfer from nano-enabled surfaces to hand was tested by wipe sampling.

- $\mathrm{Ag}$ and $\mathrm{CuO}$ nanoparticle transfer was measured by single particle ICP-MS.

- Wipe sampling allows rapid estimation for dermal exposure to nanoparticles. 